\title{
Cochlear Nucleus
}

National Cancer Institute

\section{Source}

National Cancer Institute. Cochlear Nucleus. NCI Thesaurus. Code C12837.

Located at the brainstem, this sensory org an receives auditory signals from the cochlear auditory nerve fibers. It is composed of the dorsal cochlear nucleus located on the dorsolateral surface of the inferior peduncle and the ventral (or accessory) cochlear nucleus located on the ventral aspect of the inferior peduncle. 